\title{
Popularização do autoexame da boca: um exemplo de educação não formal - PartelI
}

\author{
Popularization of oral self-examination: \\ an example of non formal education - Part II
}

Fernanda Campos Sousa de Almeida ${ }^{1}$

Dorival Pedroso da Silva ${ }^{2}$

M aria Alice Amoroso ${ }^{3}$

Reinaldo de Brito e Dias ${ }^{2}$

Oswaldo Crivello Junior ${ }^{2}$

M aria Ercilia de Araújo ${ }^{4}$

${ }^{1}$ Faculdade de O dontologia, Universidade de São Paulo.

Av. Prof. Lineu Prestes

2.227, Butantã. 05508-900

São Paulo SP.

fernandacsa@usp.br

${ }^{2}$ Departamento de Cirurgia,

Prótesee Traumatologia

M axilofaciais, Faculdadede

Odontologia, Universidade deSão Paulo.

${ }^{3} \mathrm{H}$ olding Comunicações.

${ }^{4}$ Departamento de

Odontologia Social,

Faculdade de O dontologia,

Universidadede São Paulo.
Abstract One of the largest challenges of the universities, especially the public ones, is to transpose the scientific knowledge produced into their wallsfor the general population. The non-formal education is an important tool, and yet very little used by researchers and professors to approximate the scientific knowledge. The oral cancer reaches more than 11.000 Brazilians a year. In spite of the high incidence, this pathology is still little known by the general population and part of the medical and dental class. Basing on the epidemic data in researches and scientific papers, the oral cancer was the elect theme for the action in education and communication of the first national campaign with no government character of pre vention in oral cancer, being a great example of as that can be made. This paper intends to describe the methodology used in communication and the results obtained in this successful experience. Key words Prevention, Non formal education, Oral cancer, Campaign, Communication
Resumo Um dos maiores desafios das universidades, em especial das públicas, é transpor o conhecimento científico produzido entre seus muros para a população em geral. A educação não formal éuma ferramenta importanteeainda pouco utilizada pelos pesquisadores e docentes para aproximar o cotidiano do conhecimento científico. 0 câncer de boca atinge mais 11.000 brasileiros por ano. A despeito da alta incidência, esta patologia é ainda pouco conhecida da população em geral e de parte da classe médica eodontológica. Baseando-se nos dados epidemiológicos, em pesquisas e artigos científicos, o câncer de boca foi o tema eleito para a ação em educação e comunicação da primeira campanha nacional, de caráter não governamental, de prevenção de câncer de boca, um ótimo exemplo de como isso pode ser feito. Este trabalho se propõe a descrever a metodologia de comunicação utilizada e os resultados obtidos nesta experiência.

Palavras-chave Prevenção, Educação não formal, Câncer de boca, Campanha, Comunicação 


\section{Introdução}

A comunicação éum desafio enfrentado cotidianamente por todas as pessoas, tendo em vista quea vida em sociedade exige do indivíduo capacidade mínima de estabelecer um diálogo inteligível, seja no âmbito profissional ou pessoal.

A educação, por sua vez, lança mão de ferramentas da comunicação a fim de transmitir o que se deseja ensinar e esta pode ser dividida didaticamente em três categorias. A primeira, denominada de educação formal, pode ser resumida como aquela que está presente no ensino escolar institucionalizado, cronologicamente gradual e hierarquicamente estruturado ${ }^{1}$. A segunda categoria éa educação informal, ou seja, aquela transmitida de forma espontânea e sem planejamento didático eestratégico, por pais, no convívio com amigos e parentes ou em teatros, leituras, televisão e outros meios de comunicação ${ }^{2} \mathrm{e}$, por fim, a educação não formal, que é aquela informação transmitida à população além da escola, mas a fim de complementar conteúdos da educação formal, utilizando espaços e metodologias extramuros das instituições de ensino ${ }^{3}$. Gadotti ${ }^{4}$, ainda, faz uma análise histórica sobrea educação não formal, também denominada por diversos autores como educação popular: Com as conquistas democráticas, ocorreu com a educação popular uma grandefragmentação em doissentidos: de um lado ela ganhou uma nova vitalidade no interior do Estado, diluindo-se em suas políticas públicas; e, de outro, continuou como educação não-formal, dispersando-seem milhares depequenas experiências. Perdeu em unidade, ganhou em diversidade e conseguiu atravessar numerosas fronteiras. Hoje ela incorporou-se ao pensamento pedagógico universal e orienta a atuação de muitos educadores espalhados pelo mundo [...].

Portanto, a educação não formal pode ser fruto do trabalho e da iniciativa da sociedade organizada, mas também tem grande potencial de inspirar a construção de políticas públicas, como afirma Gadotti4.

A educação em saúde pode e deve fazer uso de todas as modalidades de educação citadas para alcançar seus objetivos, ou seja, de inserir novos hábitos e mudar a opinião da população, em relação a um determinado assunto ou tema de interesse da saúde coletiva. Cada vez mais, temas como educação sexual, higiene pessoal, controle de endemias, dentre outros são trazidos por professores no ensino formal ${ }^{5}$, além de órgãos governamentais e da sociedade civil organizada, que mobilizam a opinião pública em torno de temas de interesse da saúde pública, Iançando, por vezes, mão decampanhas educativas para transmitir conceitos à população $0^{6,7}$. As campanhas contra o tabagismo do M inistério da Saúde $^{8}$ ou a bem-sucedida campanha de autoexame do câncer de mama são excelentes exemplos de estratégias que lançaram mão da educação não formal para atingir seus objetivos 9 .

Programas de educação para saúde podem fazer parte dos currículos regulares dos ensinos fundamental e médio, tratando-se, portanto, de educação formal, atingindo um universo específico de estudantes, sem falar na possibilidade desses al unos servirem de multiplicadores de informação, conceito reiterado em Brassolati e Andrade $^{10}:[$...] a escola é ponto de partida eficiente para a educação voltada à saúde pública, envolvendo di versas questões como, por exemplo, a dengue... as crianças, e especialmente os estudantes, formam classicamente um excelente canal para a introdução denovos conceitosna comunidade, pelo fato de serem membros permanentes desta [...].

A população em geral, por sua vez, para ser atingida, necessita de metodologia capaz de traduzir o conteúdo médico-científico deforma clara, em linguagem fácil eacessível, eesteéo grande desafio de acadêmicos e estudiosos da área da saúde ${ }^{11}$. U ma pergunta que inquieta a academia é como ultrapassar os muros da universidade e levar à população que a sustenta os avanços e conquistas de suas pesquisas e projetos. Como fazer extensão universitária e posicionar a academia e seus intelectuais como um grande bem da sociedade? Galassi et al. ${ }^{11}$ afirmaram que uma das respostas para essas questões está na educação não formal incorporada ao cotidiano acadêmico. 0 planejamento é o ponto-chave para o sucesso de qual quer trabalho e, em comunicação, não poderia ser diferente. Para alcançar seusobjetivos, uma campanha publicitária deve se preocupar com a escolha do tema principal e de seus pontos mais relevantes, assim como a definição do públicoalvo e da linguagem mais apropriada ${ }^{12}$.

0 câncer de boca, sexto em incidência entre homens e que atinge mais 11.000 novos brasileiros todo ano, é a mais grave doença que afeta a boca ${ }^{8}$. Lesões que necessitam de tratamentos mutilantes equeainda são detectadas tardiamente, em mais de $80 \%$ dos casos, persistem pouco conhecidas da população em geral e de parte da classe médica e odontológica.

Baseando-se nos dados epidemiológicos ena realidade do câncer de boca no Brasil, além da vocação dos pesquisadores e colaboradores do Grupo de Pesquisa em Reabilitação Maxilofacial 
(GPR), que descontentes com o pobre resultado das reabilitações em casos avançados da doença e com a pouca sobrevida de seus doentes, incluíram no escopo de suas atividades a educação em saúde e a prevenção do câncer de boca, este trabalho irá apresentar os resultados da experiência do GPR e seus membros no projeto de popularização do autoexame da boca realizado em 2005 em São Paulo.

O objetivo desta publicação é detal har a metodologia de comunicação utilizada pelo proje to, para que este possa ser reproduzido e adaptado em outros centros, e demonstrar os resultados de adesão em massa da mídia e veículos de comunicação, que possibilitaram que nossas pe ças publicitárias pudessem ser vistas além do Estado deSão Paulo, com cobertura nacional. 0 texto se preocupou em deixar claro a necessidade de planejamento em comunicação, aliando-se 0 trabalho multiprofissional com especialistas em marketing e comunicação sob supervisão e embasamento teóricos de profissionais de saúde.

\section{Material e método}

A busca por literatura e pesquisas que tratassem de campanhas sobre câncer de boca surpreendeu, tendo em vista quehavia poucos artigos disponíveis. A falta de definição de metodologia científica impede a reprodução das ações em educação ${ }^{13}$. M esmo assim, a metodologia definida pelo grupo de trabalho da campanha foi traçada com base na literatura encontrada e adaptada à realidade presente.

0 conhecimento sobre o método do autoe xame da boca, dos fatores de risco e dos principais sintomas do câncer bucal, além da necessidade de se buscar a ajuda de um profissional capacitado, quando detectadas alterações durante o autoexame, foram as informações mais importantes abordadas no momento de lançamento da I Campanha de Popularização do Autoexame da Boca, a campanha em massa na mídia. 0 foco deste trabalho foi definido como educação não formal ou educação popular ${ }^{14,15}$. A penas para propiciar ao leitor uma visão geral do que se re alizou no evento organizado pelo GPR, valemencionar a segunda etapa, que constou de atualização e capacitação dos profissionais de saúde, que receberiam a demanda por atendimento, com especial atenção à rede pública desaúde, mascom destinação de vagas a outros profissionais e acadêmicos de odontologia e medicina de faculdades parceiras.
Uma contribuição importante deste trabalho poderá ser 0 detal hamento que se segue, na me dida em que profissionais de saúde não têm intimidade com a área de marketing e comunicação; portanto, grupos que pretendam trabal har em projetos de educação popular poderão se valer das informações fornecidas sobre planejamento estratégico de comunicação utilizadas no presente artigo.

\section{Planejamento de comunicação e marketing}

Inicialmente, uma detalhada análise foi realizada com relação à situação atual do câncer deboca no Brasil e seus vários aspectos. Esta fase foi denominada de "análise estratégica", termo utilizado em comunicação para "pesquisa de mercado" 16 . Com base em dados epidemiológicos, traçou-se um perfil sociodemográfico da doença e dos pacientes acometidos pela doença, seus fatores de risco, suas formas de prevenção edetecção precoce e as possibilidades de atendimento da demanda por parte do Sistema Ú nico de Saúdee da rede privada. Algumas perguntas foram formuladas e respondidas pelas equipes de comunicação e coordenação científica. A primeira foi: qual a situação atual do câncer de boca no Brasil?

Depois de conhecer bem a doença e seus detalhes, pôde-se partir para segunda pergunta: aonde podemos chegar? 0 escopo da campanha foi divulgar o autoexame da boca principalmente, mas adicionalmente informar também sobre os fatores de risco para câncer de boca e a necessidade de buscar-se um dentista da rede básica de saúde no caso de alterações orais.

Definidos o cenário, a problemática e o que se queria alcançar, questionou-se como as metas estabelecidas poderiam ser alcançadas. A resposta para essa questão estaria na união das forças de diversos níveis da sociedade organizada, governo e universidade, em torno de ações de comunicação criativas equegerassem visibilidadepara o problema. Esta foi, portanto, a principal estratégia utilizada pelo grupo para alcançar seus objetivos.

Em comunicação e marketing, quatro pontos são de extrema importância para a definição da estratégia a ser aplicada: a matriz de potencialidade, fragilidade, oportunidade e ameaças (PFOA $)^{16}$ e os itens destacados, no caso específico da campanha, estão expostos no Quadrol.

Depossedessas informações, definiu-seo que se denomina de "formulação estratégica", na qual foi traçado o plano de trabalho para os próximos anos da campanha. U ma fase denominada 
de "crescimento" prevêa evolução da campanha nos próximos eventos e, para tanto, neste momento, um novo apelo visual, novostemas enova abordagem dos grupos-alvo da campanha estão sendo traçados para que as ações que virão possam crescer de forma sustentável.

Como meta em longo prazo, pretendemos trazer para perto órgãos internacionais a fim de que o evento se torne internacional. Esta última fase será denominada de "internacionalização".

0 período de formulação estratégica iniciase baseado em dados da fase anterior e deve contemplar a proteção do projeto, do ponto de vista jurídico. Não são raros os casos de plágios, propriedade indevida de produção intelectual. A Universidade de São Paulo prevê em seus serviços o registro de marcas e patentes eo estabelecimento de convênios a fim de proteger pesquisadores e alunos e evitar transtornos futuros, através da Agência USP de inovação (http:// www.inovacao.usp.br).

N esta fase, é possível detalhar em diversos itens todos os objetivos específicos da campanha, que neste caso foram (1) incentivar a prática do autoexame da boca; (2) influenciar as pessoas contra o tabagismo; (3) estimular os dentistas a uma melhor avaliação, de forma mais efetiva, em prol da detecção precocedo câncer de boca; (4) criar um conselho com representantes de diversos serviços do território nacional que permita e garanta a continuidade das atividades da campanha; (5) fornecer instrumentos técnicos para a capacitação de profissionais quanto ao diagnóstico das lesões; (6) sensibilizar a sociedade quanto ao problema; (7) reunir equipes multiprofissionais em torno do tema; (8) mostrar à sociedade que dentistas e médicos podem ser aliados no diagnóstico do câncer de boca; (9) atualizar profissionais nos tema de prevenção primária, prevenção secundária, tratamento oncológico e reabilitação maxilofacial; (10) produzir uma ação contínua e permanente que gere e reforce a comunicação com a população; (11) agregar celebridades que disseminem a prática do autoexame na sociedade, através da mídia; (12) trazer profissionais de renome internacional para colaborar na capacitação profissional e para possibilitar o lançamento da campanha mundial posteriormente; (13) envolver as instituições parceiras em ações de preven ção e educação em saúde e (14) estimular a pesquisa em relação às metodologias empregadas em campanhas e eventos de educação em saúde.

Além de objetivos bem definidos e pontuados, os potenciais problemas deveriam estar em mente o tempo todo e, no caso da campanha, o grande número de profissionais colaboradores de renome, as dificuldades de negociação entre

Quadro 1. Tabela de matriz de potencialidade, fragilidade, oportunidade e ameaças (PFOA).

\begin{tabular}{|l|l|}
\hline \multicolumn{1}{|c|}{ Potencialidade } & \multicolumn{1}{c|}{ Fragilidades } \\
\hline Maior evento sobre o tema no Brasil & Assunto novo e desconhecido \\
\hline Organização multidisciplinar & Muitas instituições envolvidas - potencial gerador de conflitos \\
\hline $\begin{array}{l}\text { Coordenação de uma das mais respeitadas } \\
\text { equipes de reabilitação do país }\end{array}$ & $\begin{array}{l}\text { Necessidade de agilidade nas decisões; portanto, apesar de } \\
\text { diversos colaboradores, as decisões deveriam concentrar-se } \\
\text { nas mãos de poucos (comissão representativa) }\end{array}$ \\
\hline $\begin{array}{l}\text { Chancela da USP, especialmente do Grupo } \\
\text { de Reabilitação M axilofacial (www.usp.br/ } \\
\text { fo/gpr) }\end{array}$ & $\begin{array}{l}\text { Necessidade de profissionalizar alguns setores no médio e } \\
\text { longo prazos }\end{array}$ \\
\hline $\begin{array}{l}\text { Grande apoio de instituições de renome } \\
\text { especializados }\end{array}$ & Grande lançamento sem continuidade da campanha \\
\hline $\begin{array}{l}\text { Caminhos abertos nos setores envolvidos } \\
\text { para novos projetos }\end{array}$ & $\begin{array}{l}\text { Perda do controle das ações planejadas } \\
\text { mesma intensidade }\end{array}$ \\
\hline $\begin{array}{l}\text { Carência de projetos e ações sobre o tema } \\
\text { nas políticas públicas nacionais }\end{array}$ & $\begin{array}{l}\text { Desentendimentos entre muitos colaboradores de diversas } \\
\text { áreas e com eventuais interesses divergentes }\end{array}$ \\
\hline
\end{tabular}


eles e entraves na captação de recursos foram as principais preocupações.

A próxima fase, denominada de planejamento de comunicação, contemplou todas as ações de comunicação da fase de lançamento da campanha. Para execução do plano de comunicação, foi necessário criar mecanismo de troca rápida e eficaz de informações e possibilidade de tomada de decisões rápidas diante de oportunidades e ameaças, otimizando as vantagens competitivas em relação ao ambiente. 0 planejamento foi pautado no pensamento criativo, aliado ao conhecimento científico e sempre buscando atingir me tas e objetivos traçados nas fases anteriores.

As estratégias de comunicação utilizadas foram de informação e testemunhal. A primeira prevê que a informação seja clara e precisa ao público-alvo, ou seja, a sociedade em geral e profissionais de saúde. A segunda estratégia adiciona à informação transmitida a credibilidade de celebridades, no caso da sociedade, e de personalidades do meio acadêmico, no caso de profissionais.

Como se vê, o planejamento em comunicação e educação para saúde demanda tempo e é fundamental para o sucesso das ações propriamente ditas. Passaremos, portanto, à descrição das ações realizadas nos momentos de educação realizados em maio de 2005.

\section{Educação de profissionais da saúde}

As atividades acadêmicas e científicas foram organizadas e divididas durante três dias. No primeiro, um curso gratuito de atualização profissional foi ministrado para profissionais de saúde da rede pública eestudantes de odontologia. Para facilidade, tanto de horário quanto de vagas para o curso, foi ministrado o mesmo conteúdo em doisturnos, manhã etarde, de quatro horas cada.

0 programa foi elaborado com experiências prévias da coordenação da campanha e abordou os seguintes temas: "Câncer de boca no Brasil", "Propedêutica de cabeça e pescoço", "Câncer de boca e processo de cancerização", "Exames complementares", "Reabilitação com implantes intraorais, extraorais e prótese" e, por fim, "É possível sobreviver e viver após o tratamento de câncer de boca?". Uma ficha deinscrição contendo informações aos participantes foi distribuída com auxílio deórgão da Secretaria de Saúde e da Universidade de São Paulo, devendo ser preenchida e enviada à coordenação da campanha com antecedência.

No segundo dia, foi promovido o I Fórum de Políticas Públicas em Câncer de Boca, que discu- tiu com representantes da sociedade civil organizada, universidades, terceiro setor, associações e conselhos regulamentadores e representantes de profissões e governo, assuntos concernentes ao câncer deboca e suas peculiaridades. A platéia foi composta por convidados indicados pelas entidades presentes. Além das indicações e convites, foi possível que qualquer interessado se inscrevesse desdeque solicitado convitecom antecedência à coordenação do evento. U ma pauta prévia foi enviada por e-mail aos participantes cerca de cinco dias antes do fórum, com a finalidade de facilitar discussões entrea platéia eos convidados pal estrantes. Para esta fase, os temas a serem discutidos foram divididos em prevenção primária, diagnóstico precoceclínico ehistopatológico, tratamento oncológico, reabilitação maxilofacial e comunicação em saúde. A moderação do fórum ficou a cargo do coordenador geral.

No terceiro eúltimo dia, atendendo demanda e expectativa da coordenação da campanha, iniciou-se discussão sobre procedimentos de re abilitação em cabeça e pescoço e o uso de recursos de alta complexidade. Três casos clínicos foram apresentados e os participantes e representantes de várias local idades do Brasil e entidades médicas eodontológicas discutiram dificuldades e possibilidades em reabilitar os pacientes mutilados na região de cabeça e pescoço.

\section{Educação à população em geral}

Paralelamente a uma das fases da campanha, a qual foi chamada projeto científico, foi executado um projeto de comunicação elaborado por equipe de marketing e assessoria de imprensa. Os roteiros e as definições de linguagem e dos objetivos perseguidos pelo projeto de comunicação foram elaborados pela coordenação científica da campanha.

As peças publicitárias resultado do trabalho conjunto da comunicação e da equipe científica foram um folder explicativo sobre 0 autoexame deboca, anúncios de revista sobreo tema, além de anúncios de rádio e de televisão; todos os arquivos estão disponíveis na partel desta publicação ${ }^{15}$.

Os roteiros e a linguagem utilizados na campanha e em todas as peças publicitárias foram padronizados e contemplaram a simplicidade das informações. Termos técnicos foram substituídos por outros cotidianos e optou-se por restringir a informação ao autoexame e aos dois maiores fatores de risco para câncer de boca (tabaco e álcool). As imagens utilizadas e fotos do autoexa- 
me, assim como a escolha da modelo madrinha da propaganda, contemplaram o objetivo de tornar "palatável" um assunto denso, desconhecido da mai oria e que tem uma conotação extremamente negativa na sociedade, o câncer.

0 processo deconquista deparceiros institucionais contou com esforço da equipe científicae de coordenação da campanha. Reuniões, apresentações e convites foram feitos às entidades parceiras (USP, M inistério da Saúde, CFO, CRO, $A B O$, SOBE, SBCCP, $A B C D$, Universidade Brás Cubas, dentre outras), que chancelaram as ações e deram credibilidade científica ao evento. Para sensibilização das celebridades, por outro lado, a assessoria de imprensa foi fundamental. Um intenso trabalho de convencimento foi feito efelizmente dezenas de artistas e celebridades aderiam à causa de forma voluntária e irrestrita.

Para as ações de comunicação, meios de cooperação foram estabelecidos. Dezenas de emissoras fecharam previamente e gratuitamente espaços em suas redes e garantiram a visibilidade que as ações de educação não formais necessitam para atingir a população em geral.

\section{Resultados ediscussão}

Em contraponto à realidade de diagnóstico tardio, óbitos e mutilações decorrentes do tratamento do câncer de boca, nunca houve em nosso país uma ação nacional de educação em câncer de boca, abordando de forma ampla e irrestrita suas causas, formas de prevenção e de diagnóstico precoce.

Por outro lado, muitas e importantes iniciativas regionais podem ser observadas e, mesmo com limitações de recursos e deárea de cobertu$\mathrm{ra}$, alcançam excelentes resultados. Os motivos pelo quais essas ações são isoladas e seguem padrões distintos entre si pode ser resultado de somente em 2003 terem sido traçadas metas para saúde bucal no Brasil e, até hoje, ainda não existir uma política nacional clara e organizada de abordagem do câncer de boca ${ }^{17}$. Vasconcelos ${ }^{18}$ refletiu sobre o perfil das ações de educação popular e definiu brilhantemente os movimentos de educação popular no Brasil: [...] são experiências esparsas e pontuais, sem preameaçadas desubmersão diante das constantes oposi ções: a carência de recursos das políticas sociais, a repressão política [...] profissionais comprometidos politicamente com a população não conseguem conquistar a hegemonia do seu funcionamento, mas resistem implementando pequenas práticasalternativas emar- ginais, nas quais a relação educativa com a população é priorizada.

Vale ressaltar, entretanto, que a despeito do valor local e regional dessas ações, sem divulgação científica, sem troca deinformações e experiências e sem articulação das esferas de poder, 0 impacto desses esforços na epidemiologia populacional é discutível. Especialmente quando tratamos de uma doença que necessita de atuação multiprofissional e que frequentemente é abordada com tratamentos de grande custo e complexidade, este fato pode ser comprovado pelo grandenúmero detumores sen do detectados tardiamentee deste quadro ter mudado muito pouco nos últimos anos ${ }^{19,20}$.

Outro desafio enfrentado nas universidades é diminuir o abismo que existe entre elas e a sociedade, pois existe uma fraca comunicação entre cientistas, academia esociedade, poiséuma crença popular e arraigada a de que o conhecimento científico e o desenvolvimento tecnológico são para poucos e pouco podem influenciar no cotidiano das pessoas ${ }^{21}$. 0 cientista e a universidade são vistos por parte da população de forma estereotipada e distorcida ${ }^{22}$. Sabemos que a vida moderna e globalizada é fruto do desenvolvimento da ciência e tecnologia, mas nós cientistas somos muito pouco hábeis para inserir esses conceitos e valorizar o conhecimento gerado na academia ${ }^{23}$.

0 projeto de popularização do autoexame da boca nasceu do anseio de um grupo da Universidade de São Paulo (USP), pertencente a um grupo de pesquisa, queestabel eceu em suas ações metas com relação à educação em saúde baseando-se em conceitos de transposição didática do conteúdo científico ${ }^{24}$, tendo como norte a afirmação de Antunes ${ }^{25}$ de que apenas um programa efetivo de promoção de saúde será recurso para a redução dos efeitos prejudiciais da desigualdade social sobre a saúde.

Para dar credibilidade institucional, força de atuação e penetração, o grupo pautou-se em conceitos como de Vasconcel ${ }^{26}$, que atestou ser prática comum a articulação dos grupos de educação popular com a sociedade organizada e até mesmo com o governo. Assim sendo, além do prestígio da USP, a coordenação da campanha procurou as chancelas de órgão governamentais, tais como M inistério da Saúde, secretarias estaduais e municipais, além de consel hos e associações de classes profissionais. Esses selos de qualidade foram fundamentais, mas insuficientes, tendo em vista que nenhum recurso foi alocado por parte destes grupos para o evento. Assim, 
mais uma vez ficou comprovada a falta de diretrizes públicas em câncer de boca, com destinação de esforços e verbas governamentais.

Os temas eleitos como principais para uma educação em câncer de boca foram o autoexame da boca, sendo também abordado seus principais fatores de risco, como o tabaco e o álcool ${ }^{27}$.

0 curso de atualização profissional, realizado no primeiro dia do evento, foi registrado oficialmente pela Comissão de Cultura e Extensão da Faculdade de Odontologia da Universidade de São Paulo (FOUSP) na categoria "difusão cultural". A descrição detalhada das aulas e de seus resultados podem ser encontrados na Parte I deste artigo.

0 Fórum de Políticas Públicas contou com cem pessoas, sendo 56 dessas convidadas pela coordenação do programa. Deste evento, resultou um documento com diretrizes sugeridas em câncer de boca, nos seguintes temas: (1) prevenção primária, (2) diagnóstico precoce clínico e histopatológico, (3) tratamento oncológico, (4) reabilitação maxilofacial. A partir dos temas, quatro subcomissões foram criadas com os temas discutidos e enumerados acima, contendo no mínimo quatro eno máximo oito participantes, os quais deverão se comunicar via internet e, futuramente, subsidiar discussões futuras ${ }^{28}$.

Um tema de destaque foi a urgência em se traçar políticas públicas de forma clara e com ações delongo prazo em educação em saúde. Um dos participantes afirmou que não se faz educação, não se muda conceitos ehábitos em campanhas curtas, em ocasiões e datas comemorativas, indicando a necessidade de programas permanentes de educação para que se obtenham os efeitos desejados.

Do projeto decomunicação, escopo principal deste trabalho, resultaram as peças publicitárias, que foram produzidas e pagas pelo patrocinador. Cerca de 1.800 inserções na mídia foram alcançadas em três semanas de forma gratuita; se fossem pagas, custariam cerca de $R \$ 15.000 .000,00$ (Quadro 2). Vinte e três artistas e celebridades aderiram à primeira fase do projeto. $M$ ais $R \$$ 5.000.000,00 teriam sido gastos nas mídias espontâneas, resultado da repercussão da campanha; portanto, o custo estimado deste projeto é de vinte milhões de reais.

Para se alcançar o sucesso na adesão dos meios de comunicação, alguns preceitos básicos devem ser seguidos: uma competente assessoria decomunicação devearticular os primeiros contatos com produtores e diretores de emissoras, uma postura profissional e metas bem definidas devem ser apresentadas de forma clara e objetiva, a qualidade do material de marketing é fundamental, tendo em vista que emissoras e meios de comunicação trabalham com índices de audiência, leitura, etc. eo prestígio dessas mídias está intimamente relacionado à qualidade técnica do material veiculado por elas.

0 ideal éque se estabeleçam parcerias eações cooperadas antes do evento, com definição de espaço nas mídias, período de veiculação e espaços em revistas ejornais. Vale ressaltar quea equipe de comunicação havia garantido diversos meios de comunicação, previamente acordados, para garantir o primeiro impacto da campanha, o quenaturalmenteagregaria val or eatrai ria mais mídia (mídia espontânea) evisibilidade ao evento (Quadro 2).

Uma outra estratégia fundamental éa veiculação de releases, ou seja, matérias produzidas e veiculadas para jornalistas e profissionais da área de comunicação e que geram matérias espontâneas em telejornais, rádios, revistas e jornais.

Por fim, após lançamento da campanha, foi firmado compromisso de seus idealizadores em manter a iniciativa. Uma nova logomarca, novos parceiros e patrocinadores e um projeto de longo prazo está em fase de elaboração e passa por processo de registro junto ao M inistério de Ciência e Tecnologia. Em pouco tempo, espera-se que esta iniciativa genuinamentebrasileira, nascida do sonho dedocentes ealunos daUSP eapoiada echancelada por órgãos governamentais, associações e sociedades de classes, terceiro setor, mídia e iniciativa privada, se abra a novos desafios.

\section{Considerações finais}

As campanhas, se por um lado, não formam opinião e não mudam hábitos de vida, são fundamentais para alertar a população. 0 câncer de boca e suas ações de prevenção podem seguir os passos da bem-sucedida campanha de vacinação no Brasil, quemesmo disponibilizando vacinas 0 ano todo em suas unidades de saúde, em datas préestabelecidas, une esforços econômicos e humanos e dissemina na mídia informações e convocações para a vacinação da população, facilitando assim o alcance das metas do M inistério da Saúde, com mobilização e imunização da população-alvo em massa. Fazendo um paralelo com o câncer de boca, poderíamos afirmar que, apesar de se garantir nas unidades básicas de saúde aten ção à demanda espontânea de pacientes para detecção de câncer de boca e, nes- 
Quadro 2. Tabela de mídia acordada previamente ao evento pela Holding Comunicações e Assessoria de imprensa da USP.

\begin{tabular}{|c|c|c|c|}
\hline Veículo & Tempo expos. & Total dias & $\$$ total \\
\hline IstoÉ- anúncio & Indeterminado NACIONAL & 7 & 33800 \\
\hline IstoÉ Gente & Indeterminado & 7 & 23200 \\
\hline SBT- anúncio & 6 meses/ 1 por dia NACIONAL & 182 & 4550000 \\
\hline Rádio USP & 12 spots/dia & 410 & 28700 \\
\hline Rádio J. Pan & 3 spots por 12 dias & 36 & 126000 \\
\hline TV Globo & 3 filmes/dia NACIONAL & 75 & 3375000 \\
\hline Veja São Paulo & Indeterminado & 7 & 67200 \\
\hline Rev. Pense Leve & Indeterminado & 14 & 42720 \\
\hline O Estado de SP/anúncio & Indeterminado & 1 & 76455 \\
\hline Rádio América & 8 spots/ dia por 6 meses & 216 & 86400 \\
\hline Rádio Bandeirantes & Rotativo & 18 & 45000 \\
\hline CNT & Spots/ até 05 dez & 1256 & 502400 \\
\hline Veja & IndeterminadoNACIONA & 93 & 697500 \\
\hline Produtora Oboré & 505 rádios por $8 \mathrm{~min} \mathrm{NACIONAL}$ & 505 & 35350 \\
\hline MTV/ filme & & 8 & indeterminado \\
\hline Cinemark & & 15 & 75000 \\
\hline Total parcial & & 2850 & 9764725 \\
\hline M ídia espontânea (rádio) & & spots (1256) & 502400 \\
\hline M ídia espontânea (TV) & & filmes(549) & 4117500 \\
\hline Total geral & & 4655 & 24149350 \\
\hline
\end{tabular}

Todos os valores em real ( $R$ \$) são aproximados e foram obtidos com os veículos de comunicação; assim como os tempos de exposição. M ídias "pré-estabelecidas" são aquelas "contratadas" antes do evento. M ídias espontâneas são aquelas que ocorrem nos dias do evento.

sas mesmas unidades, manter-se programas de educação continuada da população com relação aos fatores de risco, por exemplo, pode-se estabelecer datas emomentos de mobilização da opinião pública. Baseando-sena experiência do nosso grupo, este projeto de popularização mostrou que a imprensa trabalha com pautas e fatos e cabe aos profissionais de saúde pública gerar tais fatos e acontecimentos que possam concorrer com as pautas jornalísticas. Políticas públicas devem ser desenvolvidas e os profissionais envolvidos com a atenção ao paciente portador de câncer de boca devem sensi bilizar o governo e se unir em torno de uma causa, em detrimento de vaidades e atos de reserva de mercado, que pou- co contribuem para o bem maior, ou seja, a prevenção do câncer de boca esua detecção precoce.

Esta experiência piloto tem seu mérito em provar que, mesmo sem aporte financeiro governamental, o que é insatisfatório, na medida em que proporcionar saúde é um dever do Estado, que arrecada impostos e deve isso à sociedade, é possível, sensibilizando a iniciativa privada, empresários, a mídia e os profissionais das diversas áreas que compõem a equipe multidisciplinar que trabalha com câncer de boca, realizar um evento de impacto nacional, podecolaborar para o desenho de novas iniciativas que revertam o quadro temerário de incidência el evada, de morbidade e mortalidade de câncer de boca no Brasil. 


\section{Colaboradores}

FCS Almeida, DL Silva, M A Amoroso, RB Dias, O Crivello Júnior eM E Araújo participaram igualmente detodas as etapas da el aboração do artigo.

\section{Agradecimentos}

O Grupo de Pesquisa em Reabilitação M axilofacial agradece aos colegas da FOUSP, FMUSP, HCFM USP pela colaboração, às faculdades e universidades parceiras, às entidadesqueemprestaram seus prestígios ao evento, à Conexão Sistema de Prótese pelo patrocínio, à Holding Comunicação pelo brilhante trabal ho e por ser responsável por boa parte do sucesso denosso evento e aos artistas e às "mídias" pelo espaço e tempo dedicados à nossa causa.

\section{Referências}

1. Bianconi ML, Curaso F. Educação não-formal. Ciência e Cultura 2005; (4):20-23.

2. Ferrigno JC. A co-educação entre as gerações: um desafio da longevidade. Mundo saúde 1995; 29(4): 484-490.

3. Vieira VM, Bianconi L, Dias M. Espaços não-formais de ensino e currículo de ciências. Cienc. Cult. 2005; 57(4):21-23.

4. Gadotti M. Perspectivas atuais da educação. Sao Paulo Perspec. 2000; 14(2):3-11.

5. Cunha MMLC, Soares MJGO, Novo SMJ, Costa SP. Avaliação de uma proposta educativa sobre AIDS com adolescentes de escola pública de João Pessoa - PB. Rev. bras. ciênc. saúde 1998; 2(1/3):27-32.

6. Porto MP. Lutando contra a AIDS entre meninas adolescentes: os efeitos da Campanha de Carnaval de 2003 do M inistério da Saúde do Brasil. Cad Saude Publica 2005; 21(4):1234-1243.

7. Nascimento DR. A face visível da Aids. Hist. cienc. saude-M anguinhos 1997; 4(1):169-184.

8. Brasil. M inistério da saúde. [site da Internet] [acessado 2006 nov 21]. Disponível em: http:// www.inca.gov.br

9. Colley H, Hodkinson P, Malcolm J. Non-formal learning: mapping de conceptual terrain. [site da Internet] 2002 [acessado 2006 dez 30]. Disponível em: http:// www.infed.org/archives/e-texts/colley_informal_ learning.htm

10. Brassolatti RC, Andrade CFS. Avaliação de uma intervenção educativa na prevenção da dengue. Cien Saude Colet 2002; 7(2):243-251.

11. Galassi MAC, Barbin LE, Spanó JCE, M elo JAJ, Tortamano N, Carvalho ACP. Atividades extramuros como estratégia viável no processo ensinoaprendizagem. Rev. ABEN 0 2006; (1):66-69.

12. M oraes DR. AIDS, saúde reprodutiva e prevenção: estudo de caso sobre práticas educativas em uma ONG (Rio de Janeiro/Brasil). [site da Internet] 2004 [acessado 2006 dez 30]. Disponível em: http://bvssp. icict.fiocruz.br/lildbi/docsonline/get. php?id=485

13. Almeida FCS, Cazal C, Brandão TB, Araújo ME, Silva DP, Dias RB. Campanha de popularização do autoexame da boca - Universidade de São Paulo, Brasil (parte I). RBPO 2005; 4(3):147-155.

14. Belloni ML. Educação para a mídia, missão urgente da escola. Comunicação \& Sociedade 1991; 10(17): 33-46.

15. M endonça VM. Educação popular: experiências de rádio-educação no Brasil, de 1922 a 1960. Boletim Técnico do SENAC 2007; 33(1):29-41.

16. Macedo C. Comunicação Social - Publicidade e Propaganda. [site da Internet] [acessado $2006 \mathrm{dez}$ 10]. Disponível em: http://www.comunicid.com.br/ ArquivosCompartilhados/A n \% C 3\%A 1lise $\% 20$ PFOA.doc

17. Silva P. A saúde começa pela boca. [site da Internet] [acessado 2006 nov 20]. Disponível em: http:// www.usp.br/jorusp/arquivo/2005/jusp736/pag07.htm

18. Vasconcelos EM. Redefinindo as práticas de Saúde a partir de experiências de Educação Popular nos serviços de saúde. Interface (Botucatu) 2001; 8:121-126. 
19. Antunes JL, Biazevic MG, Araujo ME, Tomita NE, Chinellato LE, Narvai PC. Trends and spatial distribution of oral cancer mortality in Sao Paulo, Brazil, 1980-1998.0 ral Oncol 2001; 37(4):345-350.

20. Biazevic M G, Castellanos RA, Antunes JL, MichelCrossato $\mathrm{E}$. Trends in oral cancer mortality in the city of Sao Paulo, Brazil, 1980-2002. Cad Saude Publica 2006; 22(10):2105-2114.

21. Bazin M. Alfabetização técnica - a arte de aprender ciência e matemática. Ijuí: Editora Unijuí; 1992.

22. Lannes D, Flavoni L, M eis L. The concept of science among children of different ages and cultures. Biochemical Education 1998; 26:199-204.

23. M eis L. Ciência e educação e o conflito humano-tecnológico. São Paulo: SENAC; 2002.

24. Marandino $M$. Educational research and the production of knowledge at science museums. Hist. cienc. saude-M anquinhos 2005; 12(Suppl.):161-181.

25. Antunes JL. M ortalidade por câncer e desigualdade social em São Paulo [tese]. São Paulo (SP): Faculdade de Odontologia, Universidade de São Paulo; 2005.

26. Vasconcelos EM 2002. Educação popular e pesquisa-ação como instrumentos de reorientação da prática médica. [site da Internet] [acessado 2007 set 20]. Disponível em: http://www.anped.org.br/reunioes/ 23/textos/0620t.PDF

27. Ogden GR. Alcohol and oral cancer. Alcohol 2005; 35(3):169-173.

28. Faculdade de Odontologia de São Paulo. I Fórum de Políticas Públicas em Câncer de Boca. [site da Internet] 2005 [acessado 2008 mai 05]. Disponível em: http://www.fo.usp.br/arquivos/FORUM .pdf

Artigo apresentado em 30/03/2008

Aprovado em 30/10/2008

Versão final apresentada em 04/11/2008 